\title{
Measuring Local Thickness Through Small-Tilt Fringe Visibility
}

\author{
E. Mandell ${ }^{1}$, P. Fraundorf ${ }^{1}$, W. Qin ${ }^{2}$ \\ ${ }^{1}$ Department of Physics and Astronomy and Center for Molecular Electronics, University of Missouri-St. \\ Louis, St. Louis, MO 63121 \\ ${ }^{2}$ Advanced Products R\&D Lab, Freescale Semiconductor Inc., Chandler, AZ 85224
}

Specimen thickness measurements are often limited to analyzing one region at a time, and by the size of the electron probe. With increased availability of lattice fringe data in phase and $\mathrm{z}$ contrast images, information on how fringes change with tilt is also more accessible. We discuss how such data can provide thickness information on specimen regions only nanometers on a side, provided they are thin enough for lattice imaging. Using micrographs $1 / 3$ micron across, many regions can be analyzed with only a few images.

When tilting a thin crystalline specimen, one encounters a band of incident electron angles that give rise to visible fringes for a particular crystal lattice spacing (1). The width of this range is

$$
\alpha_{\max }=\sin ^{-1}\left[\frac{d f}{t}+\frac{\lambda}{2 d}\left(1-\left(\frac{d f}{t}\right)^{2}\right)\right] \text {, }
$$

where $\mathrm{d}$ is the lattice spacing, $\mathrm{t}$ is the crystal thickness, $\lambda$ is the electron wavelength, and $f$ is a "visibility factor" (approximately equal to 1) that accounts for the signal-to-noise ratio in detecting fringes (2). For specimens $<10[\mathrm{~nm}]$ thick and the small $\lambda$ typical of most electron microscopes, equation 1 becomes $\alpha_{\max } \cong \mathrm{d} f / \mathrm{t}$. The ensemble of all the visibility bands of a spherical crystal, oriented properly with respect to one another on a sphere, forms a fringe-visibility map $(2,3,4)$. If the visibility band half-width is $\alpha_{\max }$, the angle between the reciprocal lattice vector and the tilt axis is $\varphi$, and the total tilt range over which the fringes are visible is $\theta$, as shown in Figure 1, then Spherical Trigonometry's Law of Sines gives us

$$
\sin \left[\alpha_{\max }\right]=\sin [\varphi] \sin \left[\frac{\theta}{2}\right]
$$

Equation (2), in combination with a simplified form of equation (1), yields an expression for crystal thickness that depends on the experimentally measured quantities $\mathrm{d}, \varphi, f$ and $\theta(2)$ :

$$
t \cong(d f) \csc \left[\frac{\theta_{\text {range }}}{2}\right] \csc [\varphi] \text {. }
$$

Figures 2 and 3 show an example of two $\mathrm{TiO}_{2}$ grains from the same set of negatives taken at various tilts. The grain in Figure 2 has fringes oriented such that $\varphi=20^{\circ}$, while $\varphi=60^{\circ}$ for the grain in Figure 3 . Estimates for $\theta_{\text {range }}$ were obtained for each grain by measuring fringe intensity, and used to calculate a value for $\mathrm{t} / f$ in Angstroms. These two points are shown on Figure 4, which is a series of plots of $\mathrm{t} / f$ versus $\theta_{\text {range }}$ for different values of $\varphi$ with $d=3.5[\AA]$. Inferred thicknesses are comparable to grain widths, as expected for this set of randomly-oriented equant grains.

The primary errors come from uncertainties in $\theta_{\text {range }}$ and $f$. The variability of $f$, like the value of $f$ itself, may be investigated for a given microscope, specimen type, and operational definition for $\theta_{\text {range }}$ Errors in $f$ cause equivalent $\%$ errors in $\mathrm{t}$. Goniometer accuracy will be crucial to minimizing errors in $\theta_{\text {range }}$, even given a clear operational definition, particularly for grains with high thickness and/or $\varphi$ near $90^{\circ}$. Note from Fig. 4 that for grains with small $\varphi$ and sufficiently large tilt range that determinations of $\mathrm{t} / f$ can be quite accurate. Also note that overlapping grains may also be analyzed independently by this technique, as long as each has visible fringes, and refocusing between tilts can be done reliably.

\section{References:}

1. J. G. Allpress and J. V. Sanders, J. Appl. Cryst. 6 (1973) 165-190.

2. W. Qin and P. Fraundorf, "Cross-fringe versus single-fringe probabilities", this Proceedings (2005).

3. P. Fraundorf, W. Qin, P. Moeck and E. Mandell, arXiv:cond-mat/0212281v2 (2005).

4. W. Qin, Direct Space (nano)crystallography via HRTEM, Ph.D. thesis, University of Missouri - St. Louis/Rolla (2000). 

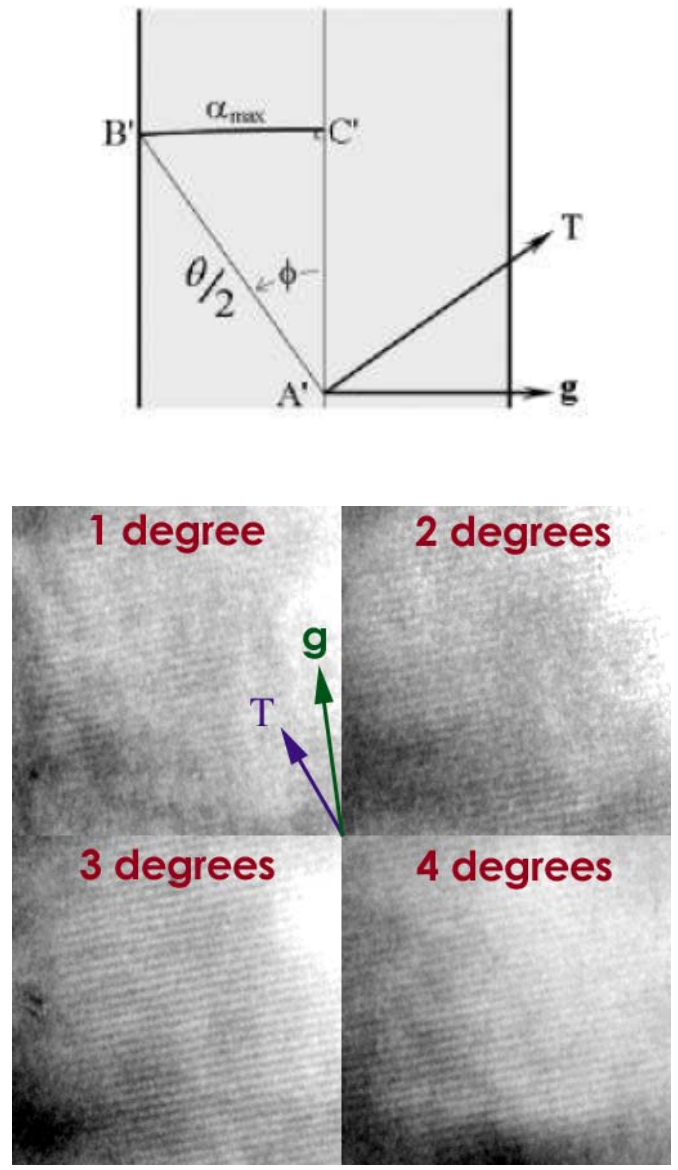

Grain 1

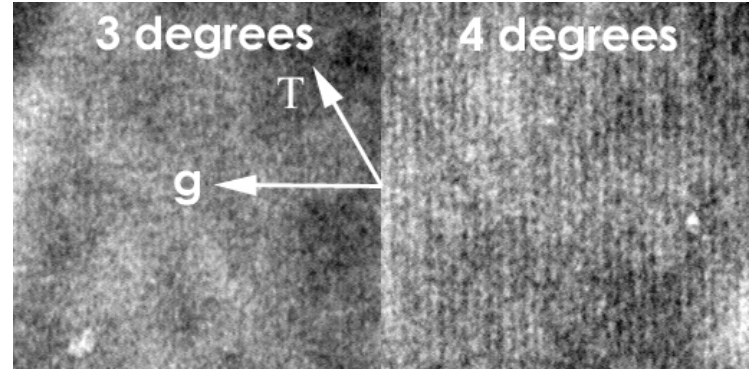

Grain 2

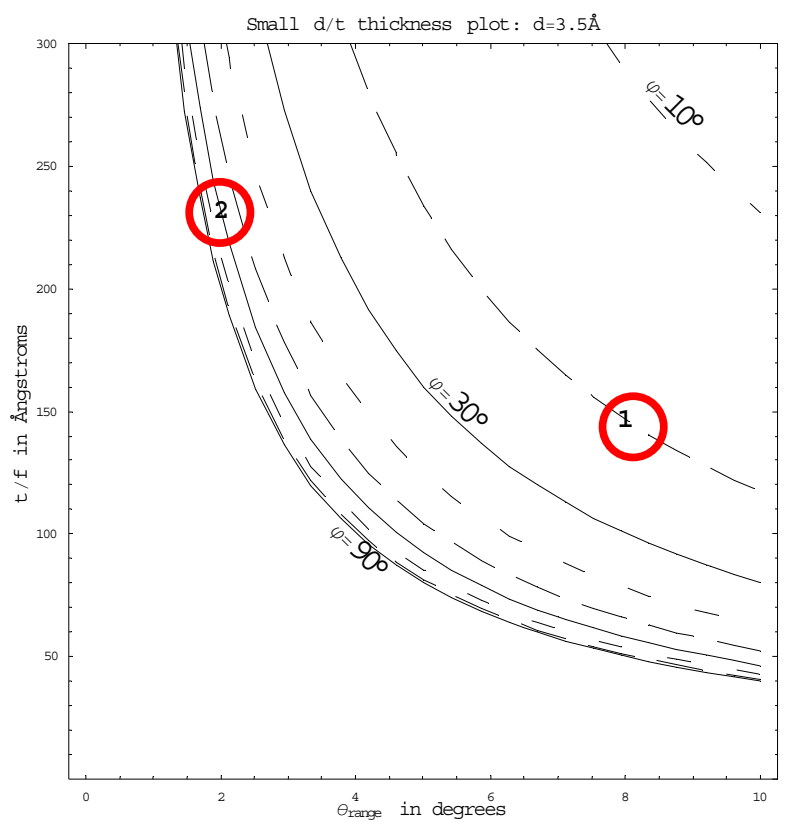

Figure 1 (upper left): A segment of a visibility band. The half-bandwidth, $\alpha_{\max }$, angle between the reciprocal lattice vector and the tilt direction, $\varphi$, and the range of tilt over which the fringes remain visible, $\theta$, allow for the determination of nanocrystal thickness.

Figure 2 (lower left): A set of image data for, grain 1, a $\mathrm{TiO}_{2}$ nanocrystal, where the reciprocal lattice vector makes a small angle with the tilt axis vector. Thus, it is expected that the fringes should be visible over a wide range of tilts. The intensity of the fringes peaks at 3 degrees.

Figure 3 (upper right): A set of image data for, grain 2, a $\mathrm{TiO}_{2}$ nanocrystal, where the reciprocal lattice vector makes a large angle with the tilt axis vector. Thus, it is expected that the fringes should be visible only over a short range tilt. The fringes are visible at 4 degrees, but practically gone at 3 degrees.

Figure 4 (lower right): A theoretical series of plots of $\mathrm{t} / f$ versus $\theta_{\text {range }}$ for different values of $\varphi$ with $\mathrm{d}=$ $3.5[\AA]$. These plots can provide a gauge of how uncertainties in $\theta_{\text {range }}$ can affect uncertainties in the measured thickness. Points 1 and 2 correspond to the estimated $\theta_{\text {range }}$ for grains 1 and 2 from experimental images taken at one-degree tilt intervals. 